not seem to apply when $n>2$. This suggests the important question: Can any fundamental circle group (discontinuous $\zeta$-group that is representable with real coefficients) be defined arithmetically as a $\Gamma_{n}$ for a sufficiently high value of $n$ ?

UNIVERSITY OF ILLINOIS, December, 1909.

\title{
A NEW ANALYTICAL EXPRESSION FOR THE NUMBER $\pi$, AND SOME HISTORICAL CONSIDERATIONS.
}

BY DR. G. VACCA.

IN the common exposition of the history of mathematics, more attention is given to results than to methods, and it is only rarely that old theorems and demonstrations are translated into the modern and living mathematical language. The great mathematicians of the eighteenth century (Euler, Lagrange, and others) have made important contributions to this difficult work. It seems to me that even to-day something can be done in this direction, and many new results can be obtained by a careful reading of the ancient classics of mathematics. The following is an example:

Let $f(x)$ be a function defined by the relation

$$
f(x)=\frac{1}{2}(x+|x|)
$$

and suppose that $f^{n}(x)$ means the result of the operation $f$ applied $n$ times to the number $x$. Then we have, if $i=\sqrt{ }-1$,

$$
{ }_{\pi}^{2}=\lim _{n=\infty} f^{n}(i) .
$$

This elegant theorem can be easily proved. It gives a possible analytical definition of the number $\pi$.

It is now to be observed that this new formula is only the analytical expression (using the geometrical representation of complex quantities of Gauss) of a series of points (approaching the vertex) of the quadratrix ( $\tau \epsilon \tau \rho a \gamma \omega \nu i \zeta o v \sigma a)$ of Deinostratos. ${ }^{*}$

We may try to transform the second member of (1) into a real expression. This can be done by elementary methods. But the result is nothing else than the well-known infinite product of complicated quadratic radicals first given by Vieta. $\dagger$

* Pappi Coll. Math., lib. IV, prop. 25.

† Vieta, Opera, ed. Schooten, Lugd. Batav., 1646, p. 400. 
But with the use of trigonometric functions the reduction of the formula (1) is immediate. We find

$$
\frac{2}{\pi}=\cos \frac{\pi}{4} \cos \frac{\pi}{8} \cos \frac{\pi}{16} \cdots
$$

an elegant expression given by Euler.*

Note. We can avoid in the formula (1) the use of the intermediate symbol $f$ by employing the notations followed by the Formulaire of Peano.

First, the parentheses in the expression $f(x)$ are useless, and were never employed by classic writers (Lagrange, Gauss).

Secondly, given an expression $A$ containing the variable $x$, it is useful to denote by $A \mid x$ the operator or functional symbol $f$ such that $f x=A$. The operation | called inversion, was first suggested by Eisenstein. $\uparrow$

Thirdly, instead of the modern symbol $|x|$ for the modulus of the quantity $x$, it is more convenient to use the classical symbol of Cauchy $\$ \bmod x$, where mod is an operator prefixed to the variable, like sin, cos, $\ldots$.

Lastly, the ordinary symbol lim has some indeterminacy : it does not express in what field the variable should vary while tending to $\infty$. A complete expression $\S$ to indicate the limiting value of the function $f$ when the variable, varying in the field $u$, tends to the value $a$ should be of the form

$$
\lim (f, u, a) \text {. }
$$

Then, if $N$ denotes the whole class of the positive integers, we have

$$
\frac{2}{\pi}=\lim \left\{\left[\left(\frac{x+\bmod x}{2} \mid x\right)^{n} i\right] n, N, \infty\right\},
$$

and this formula is complete in itself, and denotes a truth of the same kind as the formula $7+5=12$.

GenoA, Italy,

$$
\text { December, } 1909 .
$$

* Comm. Petropol., vol. 9 (1737).

† Eisenstein, Math. Abhandl., Berlin, 1847, pp. 71, 91.

$\ddagger$ Cauchy, Exercices, vol. 4 (1829), p. 47 ; Oeuvres ser. 2, vol. 9, p. 95.

$\&$ Cf. G. Peano "Sur la définition de la limite d'une fonction," American Journal of Mathematics, vol. 17 (1894). 